\title{
Tapping Mode Atomic Force Microscopy (TMAFM) of Some Multi-Component Polymers
}

\author{
Rameshwar Adhikari* \\ Central Department of Chemistry, Tribhuvan University, Kirtipur, Kathmandu, Nepal \\ Email: nepalpolymer@yahoo.com
}

\begin{abstract}
Atomic force microscopy (AFM) has been used frequently in polymer research in particular for imaging topography and phase morphology of multi-component polymers. In this work, we demonstrate the potential applications of the AFM in the study of morphology of multi-component polymers taking examples of some technically important semicrystalline polymers, blends and nanostructured block copolymers. The morphology of semicrystalline morphology could be determined ranging from molecular arrangement in the unit cells to the lamellar structure to the macroscopic morphology showing the spherulites of the polymers. Nanoscale morphology of block copolymers, nanocomposites and blends could be easily accessed by the aping mode AFM (TMAFM) phase imaging technique. It has been demonstrated that TMAFM phase imaging can be successfully utilized as a routine tool for the investigation of nanoscale morphology of the heterogeneous polymers.
\end{abstract}

Keywords: Atomic Force Microscopy (AFM), Polymer Blends, Block Copolymer, Semicrystalline Polymers

\section{Introduction}

The invention of scanning tunnelling microscopy (STM) in 1982 by Binnig and Rohrer at the IBM laboratory in Switzerland revolutionised the entire world of microscopy ${ }^{1}$. The microscope they invented was a first realisation of the quantum mechanical tunnelling principle. For the first time, an instrument could generate real space images of surfaces with atomic resolution. Within five years, this invention was awarded Nobel Prize, a clear confirmation of its importance. The STM maps the topography by measuring the tunnelling current between an atomically sharp tip and a conducting surface. Another breakthrough came in 1986 with the invention of the atomic force microscope (AFM) by Binnig, Quate and Gerber ${ }^{2}$. The AFM makes use of the measurement of the force between a sharp tip and a sample surface, and in contrast to the STM, insulating samples can also be imaged.

The schematic sketch in Fig. 1 gives an overview of the operating principle of an AFM. A sharp tip mounted on a cantilever is brought in close proximity of a surface, giving rise to the emergence of a force of interaction between the cantilever tip and the sample surface. These forces will cause the cantilever to deflect. By measuring this deflection with an optical laser deflection method (Fig. 1a), one can infer the force acting on the tip and thus also the tip-surface distance. When the tip is scanned across the surface, the tip deflection will be the result of a change in topography. Therefore, by measuring this deflection, the sample topography can be inferred (constant height mode). This, however, only works for the surfaces with very low corrugation. An alternative is to keep the force between tip and sample constant during scanning (constant force mode).

The basic principle of tapping mode AFM (TMAFM) is depicted in Fig. 1b, where two kinds of amplitudes are illustrated. When the tip subjected to free amplitude $\mathrm{A}_{0}$ comes into contact with the

\footnotetext{
* Corresponding author
} 
sample, its amplitude is drastically reduced to $A_{s p}$ (set point amplitude). The value of $r_{s p}$ (which is the ratio of set point amplitude to the free oscillation amplitude) determines the contrast in the TMAFM phase images. Quantitatively, the value of phase shift during contact (i.e., the magnitude of $\Delta \Phi$ ) determines the extent to which the local mechanical properties of the sample components differ ${ }^{3-5}$. During TMAFM phase imaging, usually, height and phase images are simultaneously collected.

During an AFM operation, the surface is scanned with an extremely sharp needle (called probe), usually shaped in a pyramidal form, attached to a cantilever. The commercially available tips usually consist of silicon $(\mathrm{Si})$ or silicon nitride $\left(\mathrm{Si}_{3} \mathrm{~N}_{4}\right)$ cantilevers micro-fabricated using photolithographic techniques. The commercially available cantilevers have different shapes (triangular or rectangular), have variable length $(100-500 \mu \mathrm{m})$ and thickness $(1-4 \mu \mathrm{m})$. The spring constant of the cantilevers varies from 0.01 to $50 \mathrm{~N} / \mathrm{m}$. The cantilevers should be flexible enough to be deflected by small forces, but also stiff enough to be withdrawn from the sample surface especially in the tapping mode operations. The radius of curvature of the tip lies typically in the range of a few nanometres ${ }^{3,6,7}$.

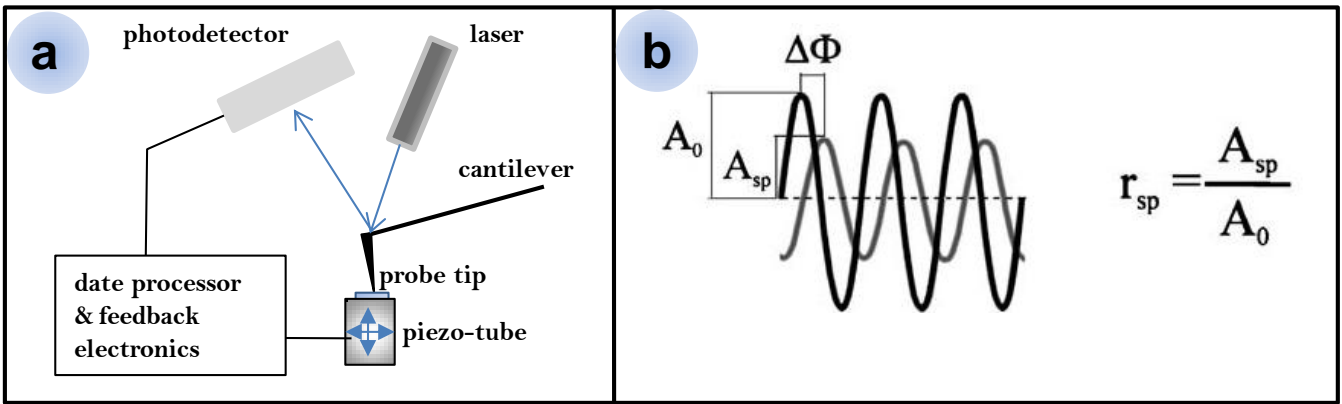

Figure 1: Schematic diagrams showing the principle of an atomic force microscope.

AFM is perhaps the most rapidly developing microscopic method. There are several operating modes of the AFM developed in the recent years. The most attractive technique widely used in polymers is the so called tapping mode. In this mode, the cantilever is allowed to swing close to its resonant frequency. The decrease in the amplitude of the resonating cantilever relative to the free oscillation and the corresponding phase shift may be recorded. The phase data are, in particular, very sensitive to the local materials properties and provide valuable information about the local chemical heterogeneity of the polymeric materials.

In the past decade, the AFM has been successfully used to gain the structural details of the semicrystalline as well as glassy polymers ${ }^{4-7}$. The AFM equipped with a local heating element has been used to study the glass transition behavior of the glassy polymers ${ }^{8}$. In addition to the imaging of lamellar structure and molecular resolution of several semicrystalline polymers ${ }^{8-11}$, real-time evolution of the morphology has been assessed ${ }^{11}$.

The aim of this paper is to study different kinds of multi-component polymers by TMAFM and demonstrate the ability of the technique to investigate a wide range of polymers and determine their morphology.

\section{Experimental Methods}

\section{Materials}

The classes of materials used in this work are listed below.

I. Semicrystalline polymers: Isotactic polypropylene (iPP) and a copolymer of iPP and atactic polypropylene (aPP) were used. Both the samples were provided by Borealis Company, Linz, Austria. 
II. Polymer blends and block copolymers: Two kinds of polymer blends were used. Semicrystalline polymer (i.e., iPP) based blends comprising nitrile rubber as dispersed phase was made available by Dr. S. Ilisch, University of Halle, Germany. Completely amorphous blends [namely acrylonitrilebutadiene-styrene (ABS) copolymer, and high impact polystyrene (HIPS)] were provided by BASF SE, Ludwigshafen, Germany. The block copolymer sample, also the amorphous polymers but forming crystal-like texture, were styrene-block-butadiene-block-styrene (SBS) triblock copolymers, and were kindly supplied by the BASF as well.

\section{Sample Preparation}

The samples were prepared by two methods: solution casting and cryo-ultramicrotomy. For solution casting, the polymer was dissolved in a suitable solvent and the polymer film was prepared by dropping the dilute solution onto freshly cleaved mica surface followed by proper solvent evaporation and annealing treatment. For ultramicrotomy, a piece of the specimen was sectioned to slices, approximately $100 \mathrm{~nm}$ thick, at $-100{ }^{\circ} \mathrm{C}$ by means of a diamond knife (Diatome, Switzerland) and an Ultramicrotome (Leica, Austria).

\section{Microscopy}

The microscopic analyses of the sample were performed on a Multimode Atomic Force Microscope (Digital Instruments, Santa Barbara, CA, USA) operated at ambient conditions operated at tapping mode. The cantilevers used were super-sharp Si micro-levers (Nanosensors, Germany) with the spring constant of $15 \mathrm{~N} / \mathrm{m}$ and tip radius of approximately $10 \mathrm{~nm}$. The resonant frequency of the cantilevers was approximately $400 \mathrm{kHz}$.

\section{Results and Discussion}

\section{Semicrystalline Polymers}

TMAFM has been used to study the morphology of several types of semicrystalline polymers ${ }^{3-11}$ which include the information ranging from molecular arrangement in the unit cells of the crystals to the crystalline arrangement in micro and mesoscale. We demonstrate here the ability of the AFM to investigate the morphology of the semicrystalline polymers in different length scales. Fig. 2 presents the AFM topography (Fig. 2, left) and derivative of the topography images (Fig. 2, right) of surface of isotactic polypropylene (iPP) film cast from its $p$-xylene solution using standard procedures. One can easily identify in real space the clearly defined typical banded spherulitic morphology of iPP in the topography and well as in the derivative images. The spherulites have diameters up to over $60 \mu \mathrm{m}$.

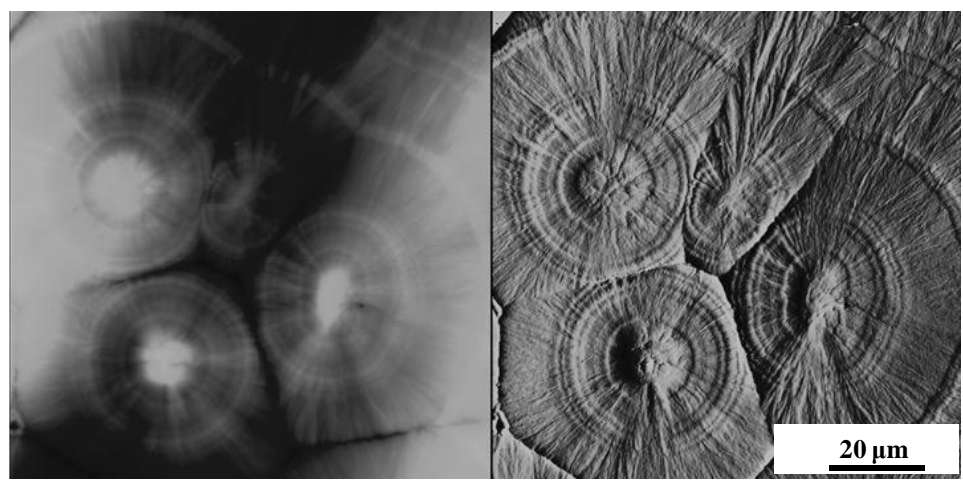

Figure 2: Topography (left) and amplitude (right) images of $\alpha$-iPP film with solution grown crystals. 
In the topography image, clearly defined gaps between the spherulites are visible which are supposed to be responsible for initiating micro-cracks upon application of external loading. The gap has been found to grow with the increase in the size of the spherulites. Thus, it has been common practice in industry to control the spherulite size in order to adjust the mechanical properties of the semicrystalline polymers.

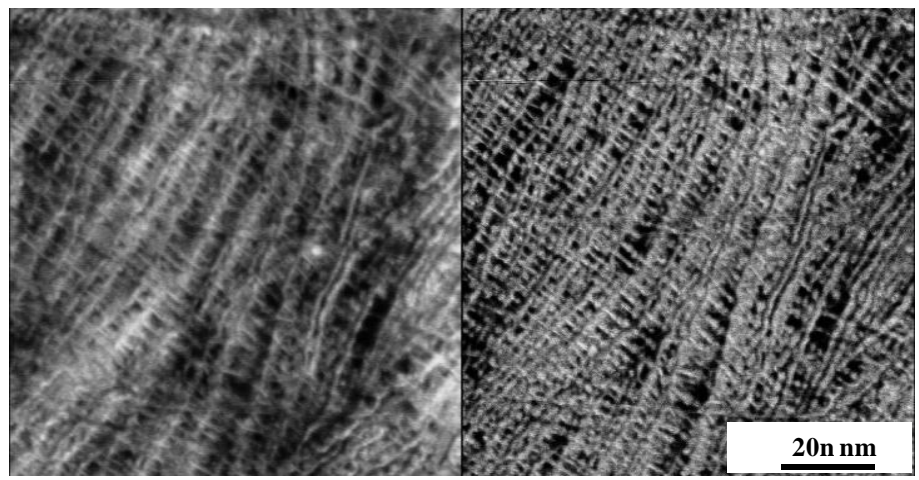

Figure 3: Tapping mode AFM images (left: topography and right: phase images) of $\alpha$-iPP showing the typical 'cross-hatched' lamellae structure, recorded under moderate tapping force.

Without changing the experimental conditions, the TMAFM can be used to record the morphology of the iPP sample down to nanoscale (see Fig. 3). Tapping mode AFM images in Fig. 3 demonstrate the lamellar morphology of $\alpha$-form of isotactic polypropylene (iPP). The radial and tangential crystalline lamellae have arranged at an angle of about $81^{\circ}$ which also corresponds to the $\beta$-angle of monoclinic unit cell of the $\alpha$ form of iPP. As a result, a typical cross-hatched morphology of the $\alpha$ - form of iPP can be observed. Provided that the surface roughness is very small, the structural details of the polymer can be easily detected both in height (Fig. 3, left) and phase signals (Fig. 3, right) under moderate tapping conditions. Both topography and phase images show practically the same structural features of the iPP sample under the application of moderate tapping force.

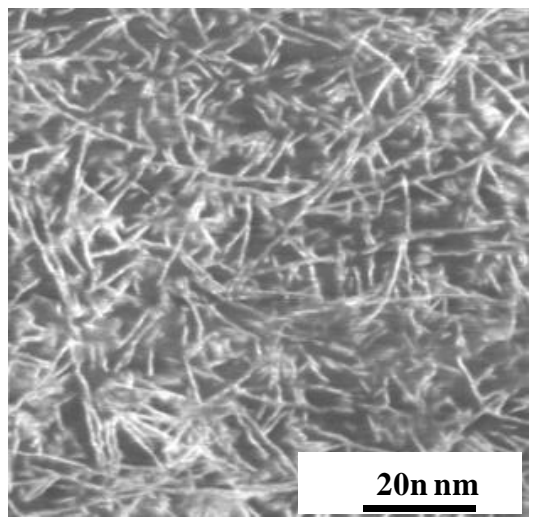

Figure 4: AFM phase image showing the morphology of a copolymer of $i P P$ and aPP; note that the cross-hatched structure of iPP (as shown in Fig.3) disappears in the copolymer.

The morphology of polypropylene actually changes dramatically with the variation in molecular structure, for instance, with tacticity of the molecules or how the methyl side groups are arranged in polypropylene macromolecule. Even iPP has several crystal modifications such as $\alpha$ form, $\beta$ form, $\gamma$ form 
etc. Syndiotactic polypropylene (sPP) has entirely different morphologies than the iPP. All these alterations have large effect on the physical properties of the polymers ${ }^{12}$.

When processed under normal conditions, the iPP, for instance, behaves as a ductile thermoplastic characterized by large elongation at break and moderately high tensile strength. Under the same processing conditions and with identical molecular weight the atactic polypropylene (aPP) would exhibit dramatically altered properties. For instance, the aPP behaves much in a manner like a rubber, i.e., has large elongation at break, shows lower tensile strength, and is characterized by large part of reversible deformation. What lies behind this change in properties is the morphology of the aPP that can be easily accessed in TMAFM phase imaging as depicted by Fig. 4 for a copolymer of iPP and aPP. In the copolymer, the ordered cross-hatched morphology of the $\alpha$-form of iPP disappears, and irregular shaped, needle like curved crystallites dispersed in the amorphous (dark appearing in Fig. 5) matrix can be observed in the phase image of the sample. These domains would orient along the deformation direction partly undergoing fragmentation processes and contribute to the elastomeric properties of the copolymer at hand.

\section{Polymer Blends and Block Copolymers}

The properties of polymers are often adjusted by combining two or more of them in different ways: physically mixing yields blends, polymerization of two or more monomers results in copolymers etc. The combinations can comprise any sorts of polymers, for instance, semicrystalline/amorphous, semicrystalline/semicrystalline and amorphous/amorphous pairs.

The first example to be presented here comprises the blend of iPP (a semicrystalline component) and nitrile rubber (an amorphous component), see Fig. 5. In this blend, iPP is a relatively stiff component while the rubber a highly flexible one. The idea of making this combination is to synergistically add only the useful properties of both the polymers for some specific applications.

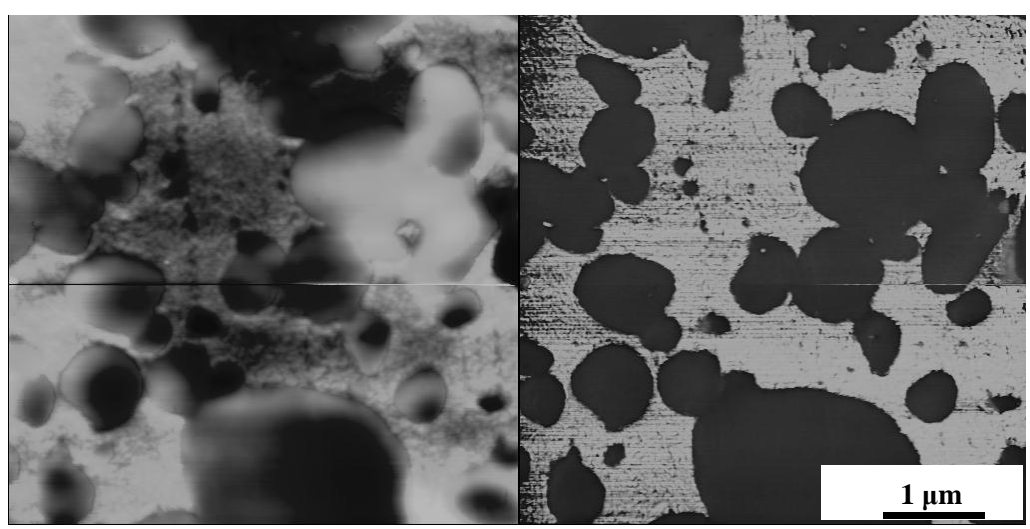

Figure 5: TMAFM topography (left) and phase (right) images of a blend of iPP and nitrile rubber as recorded from cryo-ultra-microtomed surface of the specimen.

In the topography image of the blend (Fig. 5, left), the darker areas represent the depressions while the brighter ones the elevations in the gray scale. On the phase image (Fig. 5, right) of the same sample area, the darker areas represent the rubbery phase and the brighter areas the matrix iPP. The cross crosshatched structure of the iPP matrix cannot be seen in Fig. 5 as the images are of much lower magnifications than in Fig. 3. It is very interesting to note that the excellent contrast in the phase image of the blend results due to the difference in local mechanical properties of the blend components which causes different phase shift values $\Delta \Phi$. By analyzing the phase micrograph, the dimension and size distribution of the rubber particles (the dispersed phase) can be easily evaluated. 
The results so far discussed imply that the softer polymer part appear as darker areas in the phase image and the harder one as bright area. However, this generalization is not straightforward, as the contrast in the phase image depends much on the experimental conditions ${ }^{5}$. Thus, the contrast in the AFM phase images should be cautiously interpreted. Nevertheless, without any kind of chemical treatment of the specimens, the AFM allows the analysis of local structural details of such polymers quite successfully. One can precisely estimate the local distribution and compatibility of the phases involved in heterogeneous polymers.
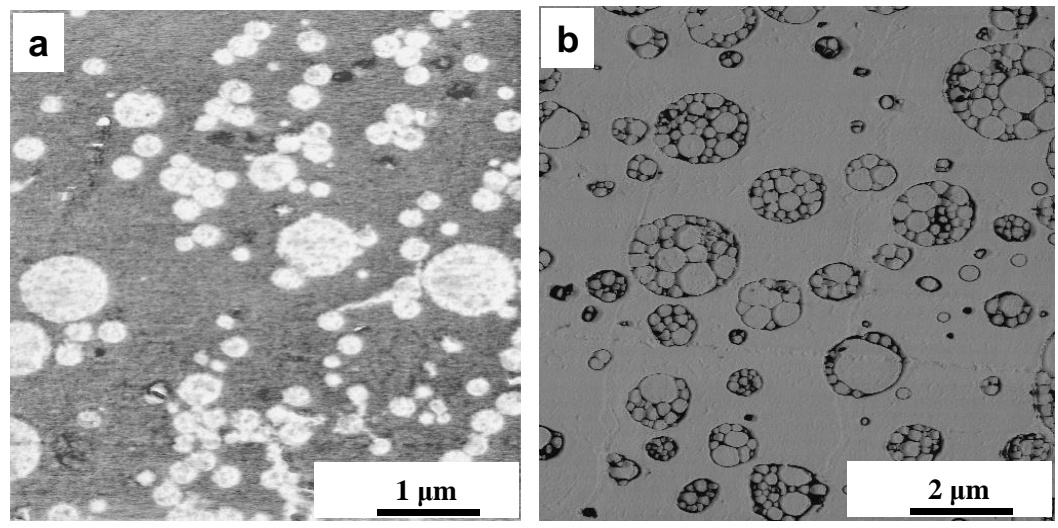

Figure 6: TMAFM phase images of polymer blends comprising hard and soft components: a) Acrylonitrile-butadiene-styrene (ABS) copolymer and b) High impact polystyrene (HIPS).

As second example, we present the TMAFM phase imaging of two different polymer blends both comprising only amorphous polymers: acrylonitrile-butadiene-styrene (ABS) copolymer and high impact polystyrene (HIPS), see Fig. 6. Both the polymers are polystyrene based and are the modified or toughened versions.

The bight spherical particles having a wide size distribution in Fig. 6a represent the stiff (or hard) polystyrene (PS) dispersed in the matrix of relatively softer acrylonitrile/butadiene copolymer. As in the previous case, the contrast arises from the differences in local mechanical properties of the components in ABS. Fig. 6b depicts the morphology of high impact polystyrene (HIPS) with typical salami structures of the dispersed particles. The HIPS comprises polystyrene and polybutadiene (PB) grafted with polystyrene (PS) chains. The blend of these components gives rise to typical morphology to the HIPS. The salami particles have wide size distribution and comprise dark appearing polybutadiene (PB) phase and bright appearing PS phase.

As the final example, we present the morphology of polystyrene-block-polybutadiene-bockpolystyrene (SBS) triblock copolymers, in which the individual chains are connected by covalent bonds. Due to inherent chemical incompatibility of the chains the chains of polystyrene (PS) and polybutadiene (PB) tend to undergo phase segregation but the extent of segregation is limited by connectivity of the molecules in a single chain. The result is the evolution of ordered nanostructures of PS and BB which can be basically controlled by adjusting the relative PS/PB composition ${ }^{13-15}$. The TMAFM can be successfully employed to image those nanostructures which were conventionally imaged by transmission electron microscopy (TEM) using chemical treatment of the ultra-thin sections of the polymers.

Fig. 7 presents phase images of two different SBS triblock copolymers having $30 \%$ (named as SBS30; Fig. 7a) and 70\% (named as SBS-70; Fig. 7b) by weight of polystyrene; the two polymers have complementary compositions. Both the polymers form periodic nanostructures with periodicity of approximately $30 \mathrm{~nm}$. Due to presence of PS as minor component, SBS-30 has bright appearing glassy PS 
phase as the dispersed component (the cylindrical domains of PS in PB matrix).
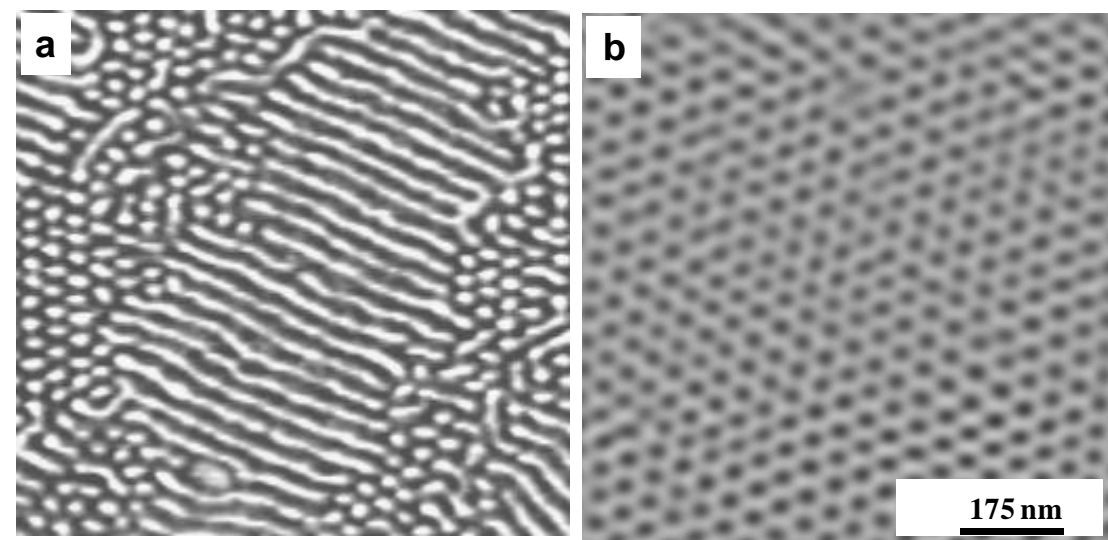

Figure 7. Phase images of different block copolymers specimens prepared by solution casting; a) SBS-30 having PS cinders in PB matrix and b) SBS-70 having PB cinders in PS matrix.

In contrary, SBS-70 has the opposite morphology (i.e., the hexagonal packed cylindrical domains of PB in PS matrix) by virtue of its composition with PS as majority component. Atomic force microscopy can be very conveniently used to image such block copolymer structures. These copolymers are important tools in nanotechnology as these can be used as templates in the manufacture of templates for various nano-objects ${ }^{13}$.

\section{Conclusions}

Different classes of polymers including semicrystalline polymer, blends and block copolymers, were investigated by tapping mode atomic force microscopy (TMAFM). It has been demonstrated that TMAFM offers an excellent tool for the determination of the polymer morphology at different length scales. Because of the possibility of the study of the materials without prior physical or chemical treatment, there is the least danger of the preparation induced artefacts in the TMAFM results of the multi-component polymers. However, care should be taken in the interpretation of the AFM data because the signals are generally very sensitive to the experimental parameters such as the tip-sample interaction force.

The AFM is one of the most reliable microscopic methods suitable for the study of the heterophase polymers which are poorly stained with conventional staining agents and/or undergo structural changes during such treatments. It is a promising technique for the study of nanostructured block copolymers, semicrystalline polymers, blends and composites.

\section{Acknowledgements}

The author acknowledges research group of Prof. Goerg H. Michler (Martin Luther University HalleWittenberg, Halle/Saale, Germany), in particular Dr. Reinhold Godehardt and Mr. Werner Lebek, for providing the laboratory facilities to conduct the AFM experiments. 


\section{References}

1. G. Binnig, C. F. Rohrer. Ch. Quate, Ch. Gerber and E. Weibel: Appl. Phys. Lett., 1982, 40, 178.

2. G. Binnig, C. F. Quate and Ch. Gerber, Phys. Rev. Lett. 1996, 56, 930.

3. H. Schönherr and G. J. Vancso, Scanning Force Microscopy of Polymers, Springer-Verlag, Berlin Heidelberg, 2010.

4. G. H. Michler, Electron Microscopy of Polymers, Springer-Verlag, Berlin Heidelberg, 2008.

5. R. Godehardt, W.Lebek, R. Adhikari, M. Rosenthal, C. Martin, S. Frangov and G. H. Michler, Eur. Polym. J., 2004, 40, 917.

6. S. N. Magonov and M.-H. Whangbo, Surface Analysis with STM and AFM, Wiley-VCH, Weinheim, 1996.

7. S. Seiko, Advances in Polymer Science 151, Springer-Verlag, Berlin Heidelberg, 2000.

8. N. Satomi, A. Takahara and T. Kajiyama, Macromolecules, 1999, 32, 4474.

9. R. Godehardt, S. Rudolf, W. Lebek, S. Goerlitz, R. Adhikari, E. Allert, J. Giesemann and G. H. Michler, J. Macromol. Sci. B, 1999, 38, 817.

10. W. Stocker, S. N. Magonov, H. J. Cantow, J. C. Wittmann and B. Lotz, Macromolecules, 1993, 26, 5915.

11. J. K. Hobbs, A. D. L. Humphris and M. J. Miles, Macromolecules, 2001, 34, 5508.

12. S. Henning, R. Adhikari and G. H. Michler, 2006, Macromol. Symp. 2004, $214,157$.

13. I. W. Hamley, The Physics of Block Copolymers, Oxford Publishers, London, 1998.

14. R. Adhikari and G. H. Michler, Prog. Polym. Sci., 2004, 29, 949.

15. I. W. Hamley, Prog. Polym. Sci., 2009, 34, 1161. 